\title{
Berberine and Prediabetes: A Clinical Observational Study
}

\author{
Li Wang1 ${ }^{*}$, Hui Ge1, Guohong Wei ${ }^{2}$, Longyun Pong³ ${ }^{3}$ Liqiu Wu' ${ }^{1}$, Huijuan Ye1 \\ ${ }^{1}$ Department of Healthcare, The First Affiliated Hospital, Sun Yat-sen University, Guangzhou, China \\ ${ }^{2}$ Department of Endocrinology, The First Affiliated Hospital, Sun Yat-sen University, Guangzhou, China \\ ${ }^{3}$ Department of Cardiology, The First Affiliated Hospital, Sun Yat-sen University, Guangzhou, China \\ Email: *wanglimer@163.com
}

How to cite this paper: Wang, L., Ge, H., Wei, G.H., Pong, L.Y., Wu, L.Q. and Ye, H.J. (2020) Berberine and Prediabetes: A Clinical Observational Study. Journal of Diabetes Mellitus, 10, 209-221. https://doi.org/10.4236/jdm.2020.104017

Received: August 23, 2020

Accepted: September 26, 2020

Published: September 29, 2020

Copyright (c) 2020 by author(s) and Scientific Research Publishing Inc. This work is licensed under the Creative Commons Attribution International License (CC BY 4.0).

http://creativecommons.org/licenses/by/4.0/

(c) (i) Open Access

\begin{abstract}
Objective: To observe the clinical efficacy and safety of berberine in treating patients with prediabetes. Methods: A total of 76 patients with prediabetes were enrolled, and at the end of a one-month run-in period, 70 patients were eligible to participate in the clinical trial and randomly assigned to two groups in a 1:1 ratio, namely a treatment group $(\mathrm{n}=35)$ and a control group $(\mathrm{n}=35)$. Members of the control group underwent lifestyle interventions, including dietary interventions and exercise prescriptions, while participants of the treatment group received both lifestyle interventions and oral administration of $300 \mathrm{mg}$ berberine, three times daily for three months. Subsequently, the treatment group entered a washout period of two months, during which time the medication was stopped, and the lifestyle interventions continued. Following the washout period, the oral administration of berberine was resumed at the same dose and lasted for three months. Blood glucose parameters were evaluated after different treatment periods. Results: 1) Intra-group comparison: Following the first and the second treatment periods, the treatment group experienced a significant decrease in fasting plasma glucose (FPG), 2-hour PG (2hPG) based on oral glucose tolerance test (OGTT), HbA1c, and HOMA-IR ( $<0.05)$; for the control group, although the blood glucose parameters exhibited a downward trend, the differences lacked statistical significance. 2) Intergroup comparison: Compared to the control group, the treatment group showed a significant decline in the levels of FPG, 2hPG, and HbAlc, whereas the decrease in HOMA-IR had no statistical significance. 3) Safety evaluation: No serious adverse events occurred in either group. Conclusion: Berberine is safe and effective in controlling blood glucose levels in patients with prediabetes and deserves to be promoted in clinical practice.
\end{abstract}




\section{Keywords}

Berberine, Diet, Exercise, Prediabetes

\section{Introduction}

Diabetes mellitus (DM) is a common metabolic disease characterized by hyperglycemia. It is caused by insulin resistance and/or insufficient insulin secretion. Hyperglycemia can lead to damage and dysfunction of target organs such as heart, brain, kidney, blood vessels and nerves. Treating diabetes and its complications impose an increasingly heavier financial burden on both patients and the government. In most cases, diabetic patients incline to focus on secondary and tertiary prevention but overlook the importance of primary prevention.

Prediabetes is considered the most significant risk factor for type 2 diabetes mellitus (T2DM). It is estimated that the world's prediabetic population will grow to 470 million by 2030 [1], and about $70 \%$ of the population will develop diabetes at some point [2]. Lifestyle modifications remain the mainstay of recommended interventions for prediabetic patients, including health education, dietary interventions, and exercise prescriptions. However, because it usually takes a relatively long time to normalize blood glucose levels, only a small number of patients can adhere to their dietary plans and exercise prescriptions.

Berberine has been used in traditional Chinese, Indian and middle-eastern folk medicine for more than 400 years. Its chemical structure as a quaternary base is quite different from other commonly used hypoglycemic agents, such as sulfonylureas, biguanides, thiazolidinediones or acarbose. It has recently been found to be a safe and effective medication that lowers blood glucose and blood lipids levels at an affordable price [3] [4]. Despite these positive findings, relevant studies on the glucose-reducing effect of berberine are largely limited to T2DM cases, without covering the population with prediabetes. Based on the traditional Chinese medicine (TCM) philosophy of corrective, preventative action against diseases, this study used berberine, in combination with lifestyle intervention, for the treatment of prediabetes, aiming to evaluate the clinical efficacy and safety of berberine in the treatment of prediabetes and provide clinical evidence for controlling T2DM with berberine as a drug intervention for primary prevention.

\section{Materials and Methods}

This study presents a randomized controlled clinical trial and has obtained approval from the hospital's Medical Ethics Committee on April 2, 2018 and written informed consent from all participants. Considering the lack of literature regarding the application of berberine on treating prediabetes, the sample size was determined by reference to a previous study using berberine for T2DM treatment [5]. 


\subsection{Inclusion Criteria}

Males or females at the age of 18 to 75 and with the fasting insulin levels (FIns) ranging from $5-20 \mathrm{mU} / \mathrm{L}$; fulfilling any of the diagnostic criteria for prediabetes introduced by the American Diabetes Association (ADA, 2010): FPG 5.6 $\mathrm{mmol} / \mathrm{L}$ to $6.9 \mathrm{mmol} / \mathrm{L}, 2 \mathrm{hPG} 7.8 \mathrm{mmol} / \mathrm{L}$ to $11.1 \mathrm{mmol} / \mathrm{L}$, and $\mathrm{HbAlc} 5.7$ to 6.4\%; no history of treatment for prediabetes; no abnormalities of thyroid function; maintaining blood pressure levels in the normal range (regardless of the use of hypertension medications); having a normal weight, or being overweight.

\subsection{Exclusion and Removal Criteria}

Exclusion criteria: patients complicated with serious diseases of the heart, brain, liver, kidneys, etc.; existing or preexisting ischemic heart disease (IHD), heart failure or stroke; large weight fluctuations of more than $3 \mathrm{~kg}$ in the recent three months; cancer; evident neurosis or mental disorder; Glucose-6-phosphate dehydrogenase(G6PD) deficiency; use of any weight-reducing aids, $\beta$-agonists (not including inhaled $\beta$-agonists), oral corticosteroids, antidepressants or antipsychotics; pregnant, nursing women or those attempting to conceive.

Removal criteria: violation of agreement, loss to follow-up or voluntary withdrawal; having severe adverse drug reactions, or serious adverse events during the clinical trial.

\subsection{General Information}

This study included 76 prediabetic patients who visited our health care, endocrinology, and cardiology clinics between January 2019 and August 2019. There was a one-month run-in period, during which all participants were provided with initial treatment consisting of dietary interventions and exercise prescriptions. Computer-tailored dietary interventions were made by determining the ideal daily intake of calories based on every participant's condition, including sugar $(50 \%-60 \%)$, fat $(20 \%-30 \%)$, and protein $(15 \%-20 \%)$ intakes; to standardize behavior modification treatment programs, standard dietary recommendations were given to all patients, who, in the meantime, were required to keep diet logs under their doctors' guidance. Two weeks later, consultant service was provided according to each patient's diet logs. Also, it was recommended that the patients increase daily physical activity and develop a regimen of aerobic exercises, i.e., jogging or running for $30 \mathrm{~min}$, five times a week. For those showing poor compliance with the exercise regimen, weight-loss behavior modifications were carried out under proper guidance. At the end of the run-in period, 70 out of the 76 patients were rendered eligible to participate in the clinical trial and were divided into a treatment group $(\mathrm{n}=35)$ and a control group $(\mathrm{n}=35)$ using a random number table.

\subsection{Treatment Methods}

The first treatment period (T1) started the next day following the end of the 
run-in period and lasted for three months. The control group received lifestyle interventions consisting of dietary control and increased physical activity, which remained the same as the regimens applied to the running period. In addition to lifestyle interventions, the treatment group was orally administrated with $300 \mathrm{mg}$ berberine according to the drug instructions (Chifeng Mysun Pharmaceutical Co., Ltd., Batch No.: 140111), three times daily with meals. Three months later, the oral administration of berberine discontinued, and a two-month washout period started, during which only lifestyle interventions were involved. The washout period was followed by the second treatment period (T2). In this period, the treatment was resumed at the same dose and lasted for three months. Weight-loss behavior modifications were introduced before and after T1 and T2, respectively.

\subsection{Observation Indicators and Methods}

Every participant was subject to preliminary screening and assessment carried out by members of the research group to collect needed information, including medical history, physical examination results, blood pressure, heart rate, body height, body weight, waistline, abdominal circumference, BMI, 12-lead ECG, liver and kidney functions, FPG, 2hPG, HbAlc, FIns and HOMA-IR, with 2hPG standing for the two-hour oral glucose tolerance test results. Ulnar vein blood was collected from the patients at around 8 to 9 a.m. after fasting for at least $8 \mathrm{~h}$, and the two-hour oral glucose tolerance test was also taken in this period. Blood samples were submitted to our central laboratory and tested using an automatic biochemical analyzer. Homeostasis model assessment of insulin resistance (HOMA-IR) was calculated according to the formula: HOMA-IR $=$ FIns $\times$ FPG/22.5 [6]. Blood glucose parameters were evaluated after T1 and T2, respectively.

\subsection{Safety Assessment}

During follow-up visits, every patient was asked a series of questions to identify whether there were any side effects or adverse reactions; diet and exercise logs were checked on a regular basis. AST and ALT levels were tested to assess liver function, while kidney function was reflected by the serum level of creatinine. All adverse events were recorded in writing.

\subsection{Statistical Analysis}

In this study, the intention-to-treat (ITT) analysis included all patients who were randomized according to randomized treatment assignment, received the experimental drug therapy at least once and participated in at least one drug efficacy evaluation. Data from T1 and T2 were found to follow non-normal distributions. Therefore, the Wilcoxon signed-rank test was employed in intra-group and intergroup comparisons, with a significance level $(\alpha)$ of 0.05 for two-tailed tests and a power $(1-\beta)$ of 0.8 . The software SPSS 26.0 was used for statistical 
analysis. Data were expressed by medians (interquartile range, IQR). Results were considered statistically significant if $\mathrm{P}<0.05$.

\section{Results}

\subsection{Patient Dropout}

After the run-in period, 70 patients were eligible for the clinical trial, among whom 67 completed the trial and the other 3 ( 2 males, 1 female) dropped out because of violation of agreement, loss to follow-up, or voluntary withdrawal. Two participants ( 1 male, 1 female) dropped out from the control group during the washout period and T2, and the dropout rate was $5.71 \%$; another participant (female) withdrew from the treatment group during $\mathrm{T} 1$, with the dropout rate of $2.86 \%$.

\subsection{Intergroup Comparison of General Information after the Run-In Period}

At the end of the run-in period, patients in the two groups showed no statistically significant differences in age distribution, male-to-female ratio, the number of cases, BMI, and blood glucose level $(\mathrm{P}>0.05)$. In other words, the two groups became broadly comparable after the run-in period. See Table 1.

\subsection{Intra-Group Comparison of Blood Glucose Parameters}

\subsubsection{FPG}

For the treatment group, the post-T1 FPG level was $5.50(5.30,5.85) \mathrm{mmol} / \mathrm{L}$, indicating a significant reduction from $6.00(5.70,6.30) \mathrm{mmol} / \mathrm{L}(\mathrm{P}<0.05)$; the FPG level was slightly increased following the washout period; at the end of T2, the FPG level was reduced from $5.60(5.40,5.77) \mathrm{mmol} / \mathrm{L}$ to $5.10(5.00,5.30)$

Table 1. Intergroup comparison of general information after the run-in period (median (IQR)).

\begin{tabular}{ccccc}
\hline Item & Control Group & Treatment Group & Z-value & P-value \\
\hline Age $(\mathrm{yr})$ & $54.00(48.50,61.50)$ & $55.00(47.50,63.50)$ & -1.023 & 0.254 \\
Percentage of females $(\%)$ & $16(45.7)$ & $16(45.7)$ & N/A & 1 \\
Height $(\mathrm{m})$ & $1.64(1.57,1.73)$ & $1.67(1.60,1.71)$ & -0.218 & 0.828 \\
Weight $(\mathrm{kg})$ & $62.98(56.03,70.48)$ & $64.94(59.46,72.69)$ & -1.075 & 0.082 \\
BMI $\left(\mathrm{kg} / \mathrm{m}^{2}\right)$ & $22.81(21.75,24.30)$ & $23.17(21.28,24.10)$ & -1.473 & 0.063 \\
FPG $(\mathrm{mmol} / \mathrm{L})$ & $6.00(5.65,6.40)$ & $6.00(5.70,6.30)$ & -0.483 & 0.629 \\
2hPG $(\mathrm{mmol} / \mathrm{L})$ & $9.10(8.40,9.95)$ & $9.00(8.10,9.70)$ & -0.882 & 0.378 \\
HbAlc $(\%)$ & $6.20(6.10,6.30)$ & $6.10(6.00,6.30)$ & -1.400 & 0.162 \\
Fins $(\mathrm{uU} / \mathrm{mL})$ & $9.53(8.27,11.00)$ & $10.35(9.23,12.73)$ & -1.556 & 0.120 \\
HOMA-IR & $2.50(2.16,2.87)$ & $2.76(2.30,3.18)$ & -1.532 & 0.125 \\
\hline
\end{tabular}

*No data was available for Fisher's z-transformation. 
$\mathrm{mmol} / \mathrm{L}(\mathrm{P}<0.05)$ (see Figure 1$)$. As to the control group, the FPG levels were respectively $6.00(5.65,6.40) \mathrm{mmol} / \mathrm{L}, 6.00(5.50,6.30) \mathrm{mmol} / \mathrm{L}, 5.90(5.22,6.20)$ $\mathrm{mmol} / \mathrm{L}$, and $5.80(5.30,6.05) \mathrm{mmol} / \mathrm{L}$ after the run-in period, $\mathrm{T} 1$, the washout period, and T2. Despite the gradual decline throughout the study, there was no significant difference in its FPG levels before and after T1 and T2.

\subsubsection{2hPG}

In the treatment group, the $2 \mathrm{hPG}$ level at the end of $\mathrm{T} 1$ was significantly decreased from $9.00(8.10,9.70) \mathrm{mmol} / \mathrm{L}$ to $6.90(6.55,7.45) \mathrm{mmol} / \mathrm{L}(\mathrm{P}<0.05)$; after the washout period, a slight increase was observed in the $2 \mathrm{hPG}$ level; the post-T2 2hPG level was lowered from the previous level of $7.30(7.15,8.20)$ $\mathrm{mmol} / \mathrm{L}$ to $6.70(6.30,7.10) \mathrm{mmol} / \mathrm{L}(\mathrm{P}<0.05)$ (see Figure 2$)$. For the control group, the $2 \mathrm{hPG}$ levels were respectively $9.10(8.40,9.95) \mathrm{mmol} / \mathrm{L}, 8.80$ (8.20, 9.40) $\mathrm{mmol} / \mathrm{L}, 8.60(8.10,9.00) \mathrm{mmol} / \mathrm{L}$ and $8.30(7.90,8.70) \mathrm{mmol} / \mathrm{L}$ following the run-in period, T1, the washout period, and T2. Although $2 \mathrm{hPG}$ was decreased gradually throughout the study, the differences before and after T1 and T2 lacked statistical significance.

\subsubsection{HbA1c}

After T1, the treatment group had an HbA1c level of $6.00(5.80,6.10) \%$, a significant reduction from the previous level of $6.10 \%(\mathrm{P}<0.05)$; the HbA1c level was slightly increased after the washout period; following T2, the HbA1c level was reduced from $6.00(5.90,6.20) \%$ to $5.80(5.70,5.90) \%(\mathrm{P}<0.05)$ (see Figure 3$)$. In the control group, the HbA1c levels were respectively $6.20(6.10,6.30) \%, 6.10$ $(6.00,6.20) \%, 6.00(5.80,6.20) \%$, and $6.00(5.80,6.10) \%$ after the run-in period,

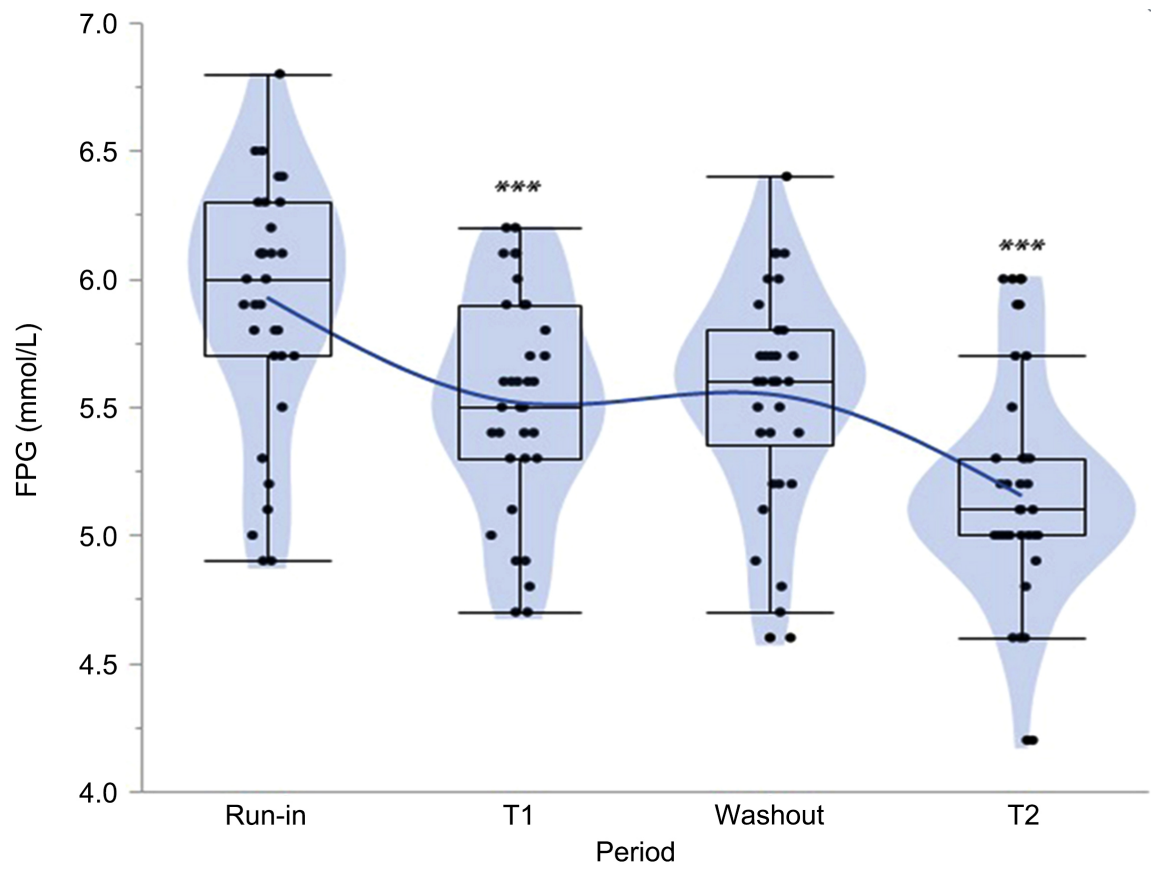

Figure 1. FPG changes in the treatment group. Note: ${ }^{* *} \mathrm{P}<0.05, \mathrm{~T} 1$ vs. Run-in and T2 vs. Washout. 


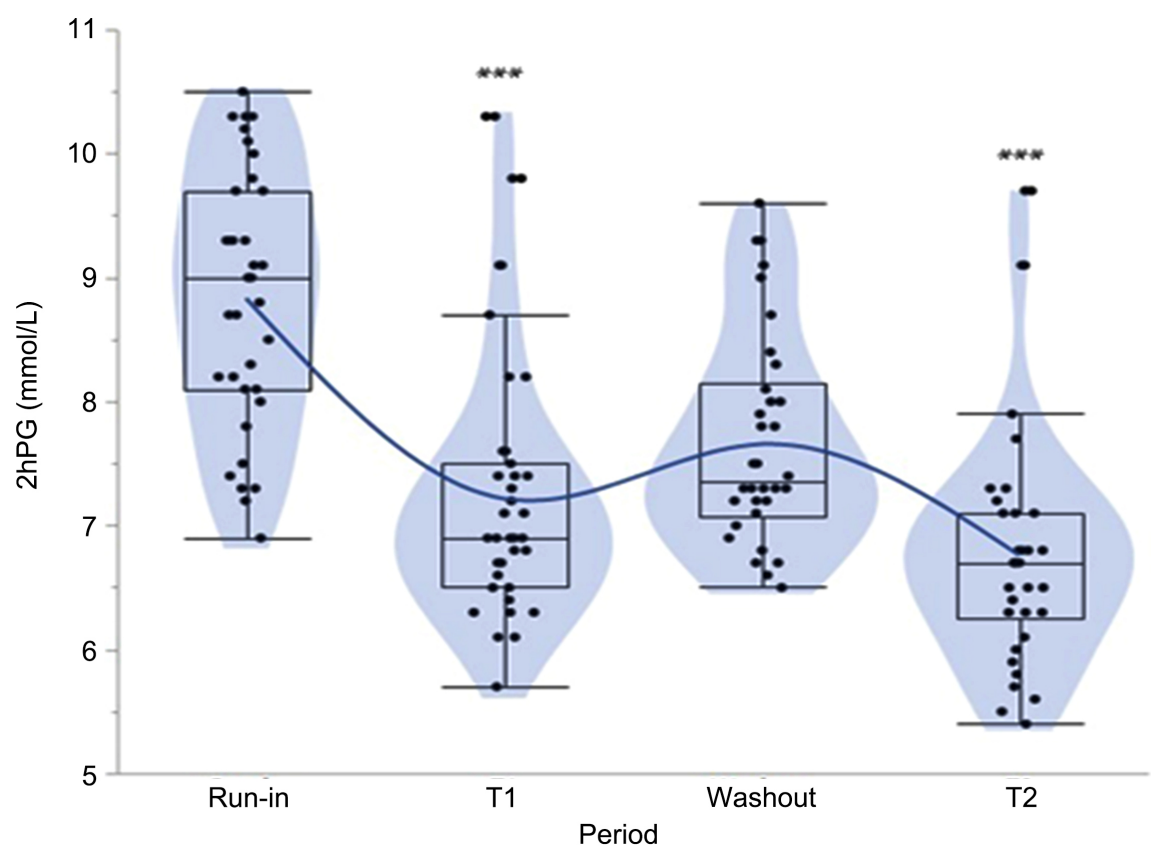

Figure 2. $2 \mathrm{hPG}$ changes in the treatment group. Note: ${ }^{\star *} \mathrm{P}<0.05, \mathrm{~T} 1$ vs. Run-in and T2 vs. Washout.

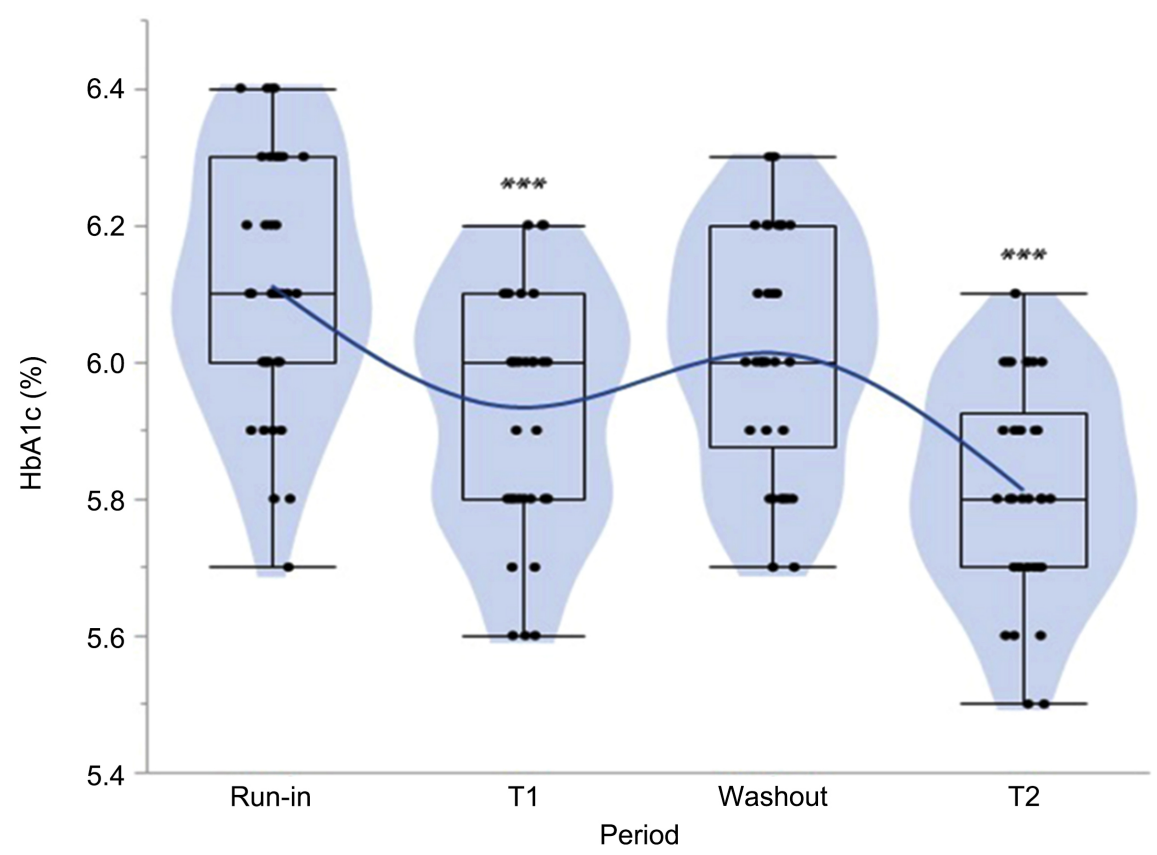

Figure 3. HbA1c changes in the treatment group. Note: ${ }^{* *} \mathrm{P}<0.05, \mathrm{~T} 1$ vs. Run-in and T2 vs. Washout.

$\mathrm{T} 1$, the washout period, and T2. Although the control group exhibited a gradual decline in its HbA1c level, there was no significant difference before and after T1 and $\mathrm{T} 2$.

\subsubsection{HOMA-IR}

In the treatment group, the post-T1 HOMA-IR level was $2.26(1.81,2.57)$, indi- 
cating a significant decrease from the previous level of $2.76(2.30,3.18)(\mathrm{P}<$ 0.05); at the end of the washout period, the HOMA-IR level rose modestly; the post-T2 HOMA-IR level was reduced to $1.88(1.62,2.22)$ from $2.37(1.94,2.77)$ $(\mathrm{P}<0.05)$ (see Figure 4). For the control group, the HOMA-IR levels were respectively $2.50(2.16,2.87), 2.33(2.02,2.66), 2.21(1.84,2.39)$ and $2.06(1.75$, 2.30) after the run-in period, T1, the washout period, and T2. The HOMA-IR level of the control group continued to decline throughout the study, but the differences before and after $\mathrm{T} 1$ and $\mathrm{T} 2$ were not statistically significant.

\subsection{Intergroup Comparison of Blood Glucose Parameters}

Compared to the control group, the treatment group showed a significant decrease in the post-T1 and -T2 FPG, 2hPG, and HbA1c levels $(\mathrm{P}<0.01)$; in terms of HOMA-IR and FIns, there was no significant difference between the control group and the treatment group (see Table 2).

\subsection{Drug Safety Evaluation}

No severe adverse event (e.g., serious liver or kidney problems, arrhythmia) occurred during the trial. There was one reported case of temporary dizziness in the treatment group during T1; a patient in the treatment group reported constipation for three days during $\mathrm{T} 1$, and the condition was relieved by oral administration of laxatives, for which reason the patient decided to drop out from the trial; another patient in the treatment group had mild diarrhea (having $1-2$ diarrhea stools daily) during $\mathrm{T} 2$, and the symptoms improved in the following two days.

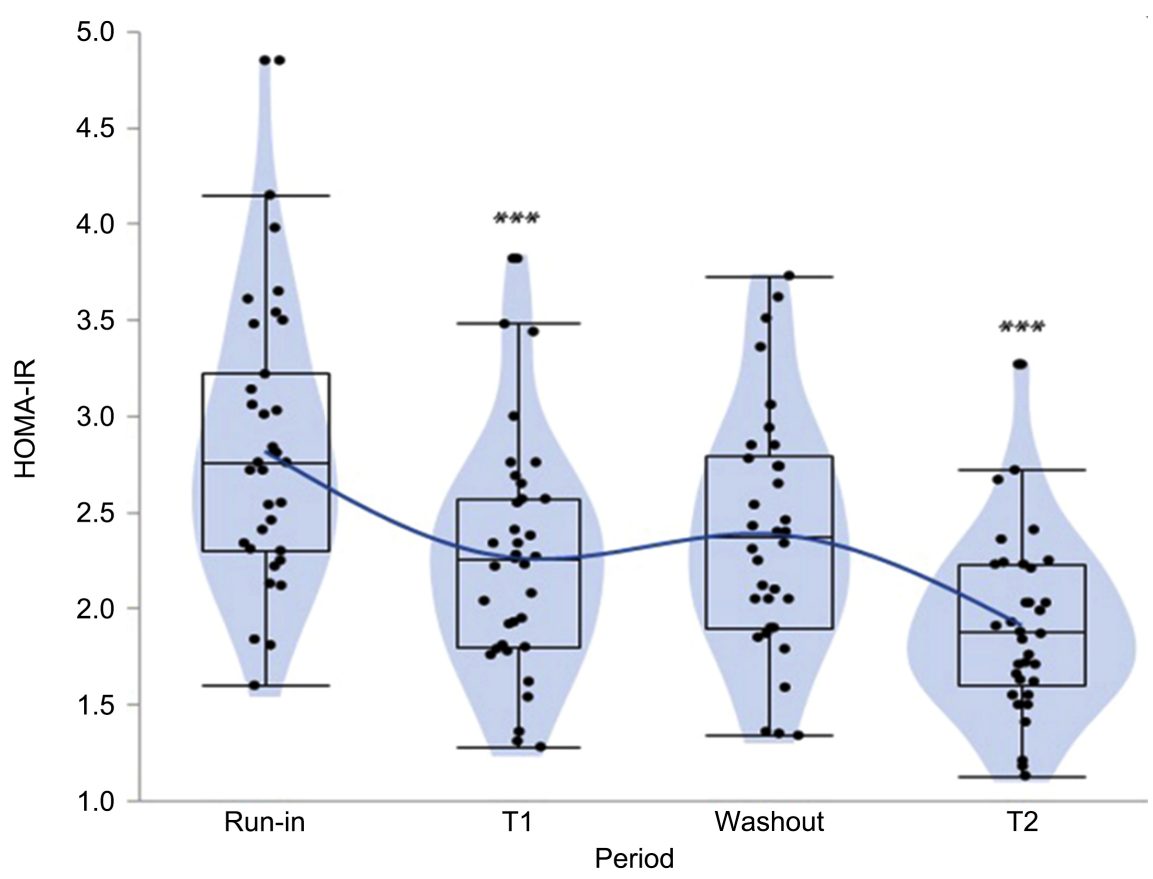

Figure 4. HOMA-IR changes in the treatment group. Note: ${ }^{\star *} \mathrm{P}<0.05$, T1 vs. Run-in and $\mathrm{T} 2$ vs. Washout. 
Table 2. Intergroup comparison of blood glucose parameters after T1 and T2 (median (IQR)).

\begin{tabular}{|c|c|c|c|c|}
\hline Item (Period) & $\begin{array}{c}\text { Control Group } \\
(\text { median }(\mathrm{IQR} \text { or } \%))\end{array}$ & $\begin{array}{c}\text { Treatment Group } \\
(\text { median }(\mathrm{IQR} \text { or } \%))\end{array}$ & $\mathrm{Z}$-value & $\mathrm{P}$-value \\
\hline FPG (run-in) & $6.00(5.65,6.40)$ & $6.00(5.70,6.30)$ & -0.483 & 0.629 \\
\hline FPG (T1) & $6.00(5.50,6.30)$ & $5.50(5.30,5.85)$ & -2.812 & 0.005 \\
\hline FPG (washout) & $5.90(5.22,6.20)$ & $5.60(5.40,5.75)$ & -1.818 & 0.056 \\
\hline FPG (T2) & $5.80(5.30,6.05)$ & $5.10(5.00,5.30)$ & -3.286 & 0.001 \\
\hline FIns (run-in) & $9.53(8.27,11.00)$ & $10.35(9.23,12.73)$ & -1.556 & 0.120 \\
\hline FIns (T1) & $9.05(7.47,10.29)$ & $8.84(7.84,10.43)$ & -0.170 & 0.865 \\
\hline FIns (washout) & $8.48(7.35,9.88)$ & $9.24(8.38,11.19)$ & -1.926 & 0.059 \\
\hline FIns (T2) & $8.22(7.11,9.26)$ & $8.27(7.35,9.25)$ & -0.135 & 0.893 \\
\hline HOMA-IR (run-in) & $2.50(2.16,2.87)$ & $2.76(2.30,3.18)$ & -1.532 & 0.125 \\
\hline HOMA-IR (T1) & $2.33(2.02,2.66)$ & $2.26(1.81,2.57)$ & -0.722 & 0.470 \\
\hline HOMA-IR (washout) & $2.21(1.84,2.39)$ & $2.37(1.94,2.77)$ & -1.257 & 0.157 \\
\hline HOMA-IR (T2) & $2.06(1.79,2.30)$ & $1.88(1.62,2.22)$ & -1.486 & 0.130 \\
\hline HbAlc (run-in) & $6.20(6.10,6.30)$ & $6.10(6.00,6.30)$ & -1.400 & 0.162 \\
\hline HbAlc (T1) & $6.10(6.00,6.20)$ & $6.00(5.80,6.10)$ & -3.983 & $<0.001$ \\
\hline HbA1c (washout) & $6.00(5.80,6.20)$ & $6.00(5.90,6.20)$ & -1.322 & 0.231 \\
\hline HbA1c (T2) & $6.00(5.80,6.10)$ & $5.80(5.70,5.90)$ & -4.348 & $<0.001$ \\
\hline 2hPG (run-in) & $9.10(8.40,9.95)$ & $9.00(8.10,9.70)$ & -0.882 & 0.378 \\
\hline 2hPG (T1) & $8.80(8.20,9.40)$ & $6.90(6.55,7.45)$ & -5.185 & $<0.001$ \\
\hline 2hPG (washout) & $8.60(8.10,9.00)$ & $7.30(7.15,8.20)$ & -2.134 & 0.057 \\
\hline 2hPG (T2) & $8.30(7.90,8.70)$ & $6.70(6.30,7.10)$ & -5.279 & $<0.001$ \\
\hline
\end{tabular}

\section{Discussion}

Prediabetes presents as impaired fasting glucose (IFG) and/or impaired glucose tolerance (IGT). A Chinese clinical study shows that every year, about 5.1\%, $11.5 \%$, and $20.2 \%$ of the individuals with IFG, IGT, and IFG + IGT shift from the prediabetic condition to diabetes [7]. The Agency for Healthcare Research and Quality (AHRQ) reported that prediabetic individuals are exposed to a significantly higher risk of developing diabetes. Annual relative risks for individuals with IFG, IGT, and IFG + IGT to develop diabetes are 6.07\% - 9.15\%, 4.35\% $4.63 \%$, and $9.96 \%-14.95 \%$, respectively [8]. At present, prediabetic patients mainly depend on lifestyle interventions to prevent or delay diabetes. If no satisfactory results are achieved after six months of active interventions, Metformin or Acarbose should be considered. For young patients having solid financial support and strong health needs, early pharmaceutical interventions are highly 
recommended [9]. Therefore, it becomes increasingly important to develop cost-effective safe hypoglycemic drugs.

Berberine (BBR, molecular formula: $\mathrm{C} 20 \mathrm{H} 19 \mathrm{NO}$, molecular weight: 353.36 ) is a natural alkaloid extracted from the rhizome of Chinese goldthread (Coptis chinensis) and Phellodendron bark (Cortex phellodendri) and is well known as an effective drug that can relieve the symptoms of infectious diarrhea. In 1988, Ni et al. first reported the hypoglycemic effect of berberine when they discovered that diabetic patients complicated with diarrhea showed a significant improvement in their blood glucose levels after using berberine [10]. Later, an in vivo study demonstrated that berberine was a promising approach for the treatment of diabetes; at that time, however, there were very few studies on prediabetes.

Prediabetes is considered an underlying etiology of metabolic syndrome and key risk factor for T2DM, cardiovascular disease, and apoplexy [11]. Insulin resistance and beta-cell dysfunction play a major role in the progression from prediabetes to diabetes [12] [13]. Although lifestyle interventions are generally considered to be safe and cost-effective, it is reported that about $10 \%-20 \%$ of the prediabetic patients are resistant to the effects of exercise with weight loss [14]. A six-year follow-up study showed that about $50 \%$ of the prediabetic patients who had received lifestyle interventions still developed diabetes [15]. This study investigated the clinical efficacy of berberine in treating prediabetes based on lifestyle interventions. To minimize the effects of confounding factors, the clinical trial included a run-in period and a washout period. The intra- and inter-group comparisons showed that berberine could significantly reduce the levels of FPG, $2 \mathrm{hPG}$, and $\mathrm{HbA1c}$ in patients with prediabetes; in the treatment group, HOMA-IR became lower than the pretreatment level after the use of berberine; the hypoglycemic effects of berberine in $\mathrm{T} 1$ and $\mathrm{T} 2$ period were similar. All this indicates that berberine contributes to lower blood glucose levels and improved insulin resistance in prediabetes, which shows even better efficacy when in combination with lifestyle interventions. In a meta-analysis of berberine-based treatment of T2DM, compared to the control group (subject to either placebo or non-pharmaceutical intervention), berberine could significantly reduce such blood glucose parameters as FBG, PBG, HbA1c, and HOMA-IR [16], which agrees with the results of this study. Noteworthily, berberine may become less effective as a hypoglycemic drug when a patient is older than 60 , the daily dosage exceeds $2 \mathrm{~g}$, or the course of treatment lasts longer than 90 days [17]. Metformin (MET) is recognized as a basic hypoglycemic drug. Subgroups analysis of berberine compared with MET showed that $1.5 \mathrm{~g} / \mathrm{d}$ MET was significantly better than berberine $(0.9-1.5 \mathrm{~g} / \mathrm{d})$ in lowering FBG and PBG. However, there was no significant difference between $1.5 \mathrm{~g} / \mathrm{d}$ berberine and $0.75 \mathrm{~g} / \mathrm{d}$ MET groups in blood glucose profiles [16].

Although the hypoglycemic effect of berberine has been demonstrated at the cellular and molecular level, as well as in animal models, the underlying mechanism is still not fully understood. It is believed that BBR-mediated activation 
of AMP-activated protein kinase (AMPK) plays a critical role in its hypoglycemic effect because the active form of AMPK is able to stimulate glucose and fatty acid oxidation in cells and improve insulin sensitivity [18]. With diabetes being a low-grade inflammatory disease, berberine inhibits NF- $\kappa \mathrm{B}$ and proinflammatory factors, thereby controlling oxidative stress and inflammation [19]. Gluconeogenesis mainly occurs in the liver, and berberine manages to improve blood glucose metabolism by inhibiting liver gluconeogenesis in diabetic rats via the LKB1/AMPK/TORC2 signaling pathway [20]. Besides, it is found that berberine can inhibit the activity of $\alpha$-glucosidase in the small intestine and reduce digestion and absorption of glucose in the intestinal tract [21], in which case it produces a hypoglycemic effect as well as the first-line drug Acarbose.

In this study, no serious adverse events but mild, temporary dizziness or gastrointestinal discomfort occurred in very few patients. In one of our previous studies, our research group prescribed berberine for normoglycemia combined with mild hyperlipemia and discovered that berberine could lower blood lipid levels without causing hypoglycemia [22]. This indicates that berberine is safe to use and has no obvious side effects.

This study has a few limitations. First, it is not a blind design; it is a single-center design with a small sample size and non-normally distributed data; no classification of prediabetes is provided even though there are matrix differences, and as a result, berberine may be overrated with regard to its clinical efficacy and safety. Second, because failed lifestyle interventions are not discussed in this study, it does not provide any insights into whether or not berberine is effective in treating prediabetes without lifestyle interventions. Thus, multicenter clinical trials with a larger sample size are needed to confirm the results of this study and clarify the uncertainties.

\section{Conclusion}

This study indicates that berberine is effective in controlling FPG, $2 \mathrm{hPG}$ and $\mathrm{HbA} 1 \mathrm{c}$ and improving insulin resistance in patients with prediabetes. It can delay or even prevent the transition from prediabetes to T2DM. Berberine is a safe drug with no obvious side effects. It is especially cost-effective when combined with lifestyle interventions, which deserves to be promoted in clinical practice. However, better large-scale randomized controlled clinical trials (RCTs) are needed to further verify the therapeutic effects of berberine.

\section{Funding}

This research was supported by a grant from the Project of Administration of Traditional Chinese Medicine of Guangdong Province of China (Grant No.: 20191065).

\section{Conflicts of Interest}

None of the authors has any potential conflicts of interest associated with this research. 


\section{References}

[1] Glauber, H., Vollmer, W. and Nichols, G. (2018) A Simple Model for Predicting Two-Year Risk of Diabetes Development in Individuals with Prediabetes. The Permanente Journal, 22, 17-50.

[2] Bigna, J., Nansseu, J., Katte, C. and Noubiap, J.J. (2018) Prevalence of Prediabetes and Diabetes Mellitus among Adults Residing in Cameroon: A Systematic Review and Meta-Analysis. Diabetes Research and Clinical Practice, 137, 109-118. https://doi.org/10.1016/j.diabres.2017.12.005

[3] Wang, H.J. and Chiang, B.H. (2012) Anti-Diabetic Effects of a Traditional Chinese Medicine Formula. Food \& Function, 3, 1161-1169. https://doi.org/10.1039/c2fo30139c

[4] Dong, H., Zhao, Y., Zhao, L. and Lu, F.E. (2013) The Effects of Berberine on Blood Lipids: A Systemic Review and Meta-Analysis of Randomized Controlled Trials. Planta Medica, 79, 437-446. https://doi.org/10.1055/s-0032-1328321

[5] Yin, J., Xing, H. and Ye, J. (2008) Efficacy of Berberine in Patients with Type 2 Diabetes Mellitus. Metabolism Clinical and Experimental, 57, 712-717. https://doi.org/10.1016/j.metabol.2008.01.013

[6] Matthews, D.R., Hosker, J.P., Rudenski, A.S., Naylor, B.A., Treacher, D.F. and Turner, R.C. (1985) Homeostasis Model Assessment: Insulin Resistance and Beta-Cell Function from Fasting Plasma Glucose and Insulin Concentrations in Man. Diabetologia, 28, 412-419. https://doi.org/10.1007/BF00280883

[7] Yang, W.Y., Lu, J.N., Weng, J.P., et al. (2010) Prevalence of Diabetes among Men and Women in China. The New England Journal of Medicine, 362, 1090-1101. https://doi.org/10.1056/NEJMoa0908292

[8] Xu, H.N. (2016) Compare National and International Guidelines for Pre-Diabetes Population Management. Diabetes World, 10, 61-64.

[9] China Pre-Diabetes Clinical Intervention Expert Group (2019) Chinese Experts Consensus of Clinical Intervention for Pre-Diabetes (Draft for Comments). Drug Evaluation, 16, 3-16.

[10] Ni, Y.X. (1988) Therapeutic Effect of Berberine on 60 Patients with Type II Diabetes Mellitus and Experimental Research. Chinese Journal of Integrative Medicine, 8, 711-713.

[11] Weiss, R., Santoro, N., Giannini, C., Galderisi, A., Umano, G.R. and Caprio, S. (2017) Prediabetes in Youths: Mechanisms and Biomarkers. The Lancet Child \& Adolescent Health, 1, 240-248. https://doi.org/10.1016/S2352-4642(17)30044-5

[12] Kim, C., Kim, H., Kim, E., Bae, S.-J., Choe, J. and Park, J.-Y. (2018) Longitudinal Changes in Insulin Resistance, Beta-Cell Function and Glucose Regulation Status in Prediabetes. The American Journal of the Medical Sciences, 355, 54-60. https://doi.org/10.1016/j.amjms.2017.09.010

[13] Kabadi, U. (2017) Major Pathophysiology in Prediabetes and Type 2 Diabetes: Decreased Insulin in Lean and Insulin Resistance in Obese. Journal of Endocrinology, 1, 742-750. https://doi.org/10.1210/js.2016-1116

[14] Malin, S.K., Liu, Z., Barrett, E.J., et al. (2016) Exercise Resistance across the Prediabetes Phenotypes: Impact on Insulin Sensitivity and Substrate Metabolism. Reviews in Endocrine and Metabolic Disorders, 17, 81-90. https://doi.org/10.1007/s11154-016-9352-5

[15] Pan, X.R., Li, G.W., Hu, Y.H., et al. (1995) Effect of Dietary And/Or Exercise Intervention on Incidence of Diabetes in 530 Subjects with Impaired Glucose Tolerance 
from 1986-1992. Chinese Journal of Internal Medicine, 34, 108-112.

[16] Wei, X.C., Zhu, L.Q. and Wang, C. (2015) Efficacy and Safety of Berberine in Patients with Type 2 Diabetes Mellitus: A Meta-Analysis. Chinese Herbal Medicine, 7, 344-353. https://doi.org/10.1016/S1674-6384(15)60063-6

[17] Liang, Y.P., Xu, X.J., Yin, M.J., et al. (2019) Effects of Berberine on Blood Glucose in Patients with Type 2 Diabetes Mellitus: A Systematic Literature Review and a Meta-Analysis. Endocrine Journal, 66, 51-63. https://doi.org/10.1507/endocrj.EJ18-0109

[18] Lee, Y.S., Kim, W.S., Kim, K.H., et al. (2006) Berberine, a Natural Plant Product, Activates AMP-Activated Protein Kinase with Beneficial Metabolic Effects in Diabetic and Insulin-Resistant States. Diabetes, 55, 2256-2264.

https://doi.org/10.2337/db06-0006

[19] Jiang, Q., Liu, P., Wu, X., et al. (2011) Berberine Attenuates Lipopolysaccharide-Induced Extracelluar Matrix Accumulation and Inflammation in Rat Mesangial Cells: Involvement of NF-KB Signaling Pathway. Molecular and Cellular Endocrinology, 331, 34-40. https://doi.org/10.1016/j.mce.2010.07.023

[20] Jiang, S.J., Dong, H., Li, J.B., et al. (2015) Berberine Inhibits Hepatic Gluconeogenesis via the LKB1-AMPK-Torc2 Signaling Pathway in Streptozotocin-Induced Diabetic Rats. World Journal of Gastroenterology, 21, 7777-7785.

https://doi.org/10.3748/wjg.v21.i25.7777

[21] Pan, G.Y., Huang, Z.J., Wang, G.J., et al. (2003) The Antihyperglycaemic Activity of Berberine Arises from a Decrease of Glucose Absorption. Planta Medica, 69, 632-636. https://doi.org/10.1055/s-2003-41121

[22] Wang, L., Peng, L.Y., Wei, G.H., et al. (2016) Therapeutic Effects of Berberine Capsule on Patients with Mild Hyperlipidemia. Chinese Journal of Integrated Traditional and Western Medicine, 36, 681-684. 